\title{
Novel NOTCH1 mutations in patients with bicuspid aortic valve disease and thoracic aortic aneurysms
}

\author{
Stephen H. McKellar, MD, ${ }^{a, *}$ David J. Tester, BS, ${ }^{b}$ Marineh Yagubyan, MD, ${ }^{\text {a }}$ Ramanath Majumdar, $\mathrm{PhD},{ }^{a}$
} Michael J. Ackerman, MD, PhD, ${ }^{b}$ and Thoralf M. Sundt III, MD

From the Department of Surgery/Division of Cardiovascular Surgery, and the Departments of Medicine, Pediatrics, and Molecular Pharmacology and Experimental Therapeutics/Divisions of Cardiovascular Diseases and Pediatric Cardiology, ${ }^{\mathrm{b}}$ Mayo Clinic College of Medicine, Rochester, Minn.

Financial support from SS-50 Grant (Mayo Clinic, award recipient Dr T. M. Sundt) and Sullivan Foundation is gratefully acknowledged. This work was supported by the Mayo Clinic Windland Smith Rice Comprehensive Sudden Cardiac Death Program. M.J.A. is an Established Investigator with the American Heart Association and is the director of the Mayo Clinic Windland Smith Rice Sudden Death Genomics Laboratory.

Received for publication Oct 24, 2006; revisions received Jan 22, 2007; accepted for publication Feb 15, 2007.

Address for reprints: Thoralf M. Sundt III, MD, Division of Cardiovascular Surgery, Mayo Clinic, 200 First St SW, Rochester, MN 55905 (E-mail: sundt.thoralf@mayo. edu).

*Clinician Investigator Program, Mayo School of Graduate Medical Education, Mayo Clinic College of Medicine.

J Thorac Cardiovasc Surg 2007;134:290-6

$0022-5223 / \$ 32.00$

Copyright $\odot 2007$ by The American Association for Thoracic Surgery

doi:10.1016/j.jtcvs.2007.02.041
Objectives: Bicuspid aortic valve is a common condition and is associated with a significantly increased risk of developing thoracic aortic aneurysms and acute aortic dissection. Patient-specific prediction of the risk of developing thoracic aortic aneurysm, however, is imprecise. We hypothesize that genotypic variations in patients with bicuspid aortic valves contribute to this observed variability in aortic phenotype. We, therefore, investigated the potential relationship between mutations in regions of $\mathrm{NOTCH} 1$ recently reported to be associated with bicuspid aortic valve and the phenotype of bicuspid aortic valve and thoracic aortic aneurysms in unrelated patients undergoing surgical repair.

Methods: We performed a targeted mutational analysis of $\mathrm{NOTCHI}$ using genomic DNA from 48 unrelated subjects with concomitant bicuspid aortic valve and thoracic aortic aneurysm using denaturing high-performance liquid chromatography and DNA sequencing. We focused on exons in which mutations associated with bicuspid aortic valve have been reported previously. Results were compared with control subjects with trileaflet aortic valves $(\mathrm{n}=94)$, bicuspid aortic valves, and normal aortas $(\mathrm{n}=22)$ and in subjects with tricuspid aortic valves and thoracic aortic aneurysms $(\mathrm{n}=28)$.

Results: Four unique, nonsynonymous (3 novel) variants were identified in 5 $(10.4 \%)$ of 48 patients with concomitant bicuspid aortic valves and thoracic aortic aneurysms compared with only $3(2.1 \%)$ of 144 control subjects $(P=.02)$. Of these, 2 novel missense mutations, A1343V and P1390T, were observed only in patients with bicuspid aortic valves and tricuspid aortic aneurysms.

Conclusions: This targeted analysis involving NOTCH1 exons previously implicated in familial and sporadic bicuspid aortic valve demonstrates overrepresentation of NOTCHI missense variants among patients with bicuspid aortic valves and thoracic aortic aneurysms. Identification of aneurysm-predisposing susceptibility genes may lead to gene-directed surgical therapy of the ascending aorta for patients with bicuspid aortic valves.

B icuspid aortic valve (BAV) disease is the most common congenital cardiovascular malformation and is responsible for a large proportion of patients coming to aortic valve replacement (AVR). ${ }^{1}$ Individuals with BAV are at increased risk of developing thoracic aortic aneurysms (TAA), acute aortic dissection, and premature death relative to the normal population ${ }^{2}$ and have a demonstrably higher incidence of postoperative aortic dissection after AVR. ${ }^{3}$ Accordingly, some surgeons advocate prophylactic replacement of an "even seemingly normal" ascending aorta at the time of AVR for BAV. ${ }^{3}$ Aneurysmal disease does not develop in all patients with BAV, however, and such an approach would needlessly subject a large number of patients to a procedure with a predictably higher operative risk. ${ }^{4}$ Clinical risk stratification, however, is imprecise. 


$$
\begin{aligned}
& \text { Abbreviations and Acronyms } \\
& \text { AVR = aortic valve replacement } \\
& \text { BAV = bicuspid aortic valve } \\
& \text { DHPLC = denaturing high-performance liquid } \\
& \text { chromatography } \\
& \text { PCR = polymerase chain reaction } \\
& \text { TAA = thoracic aortic aneurysm } \\
& \text { TAV = trileaflet aortic valve } \\
& \text { TGF = transforming growth factor }
\end{aligned}
$$

Both TAA disease and BAV disease are inheritable disorders in at least some cases. Familial clustering of BAV disease shown by Clementi, ${ }^{5}$ Cripe,${ }^{6}$ and their colleagues has demonstrated heritability $\left(\mathrm{h}^{2}\right)$ of $89 \%$ with autosomal dominant transmission and incomplete penetrance of the condition. In addition to Marfan syndrome and the more recently identified Loeys-Dietz syndrome, ${ }^{7}$ a genetic basis for some cases of familial TAA and dissection has been mapped by Milewicz, Basson, and their colleagues to 3 genetic loci: 5q13-14, ${ }^{8} 11 \mathrm{q} 23,{ }^{9}$ and $3 \mathrm{p} 24-25,{ }^{10,11}$ the last of which has proven to be the transforming growth factor (TGF) $\beta$-2 receptor.

Recently, an association between mutations in $\mathrm{NOTCHI}$ and aortic valve disease has been described. NOTCH1 encodes for a transmembrane protein that activates a signaling pathway with an active role in cardiac embryogenesis, including aortic and pulmonary valve development as well as the development and maintenance of the aorta and other great vessels. ${ }^{12-15}$ Garg and colleagues ${ }^{16}$ reported NOTCH1 mutations in 2 pedigrees with an assortment of cardiovascular disease phenotypes, including BAV. Subsequently, Mohamed and coworkers ${ }^{17}$ reported 2 mutations in different exons of the same gene in patients with nonfamilial BAV; however, the status of the aorta was not described in the former and only briefly mentioned in the latter study. To date, no studies have identified genetic mutations common to both BAV and TAA phenotypes.

Recent advances in the field of genomics introduce the possibility of genetic profiling as is currently used in pharmacogenomics. Identification of genes predictive of TAA in patients with BAV may foster "gene-directed" surgical therapy of the ascending aorta. We hypothesize that genetic variability among patients with BAV may explain the phenotypic variability in the subset of patients with BAV in whom TAA develops. We, therefore, performed a targeted mutational analysis of NOTCH1 from genomic DNA obtained from patients with BAV and TAA using denaturing high-performance liquid chromatography (DHPLC) and direct DNA sequencing. These results were compared with DNA from 188 reference alleles obtained from control subjects $(\mathrm{n}=94)$ with normal, trileaflet aortic valves

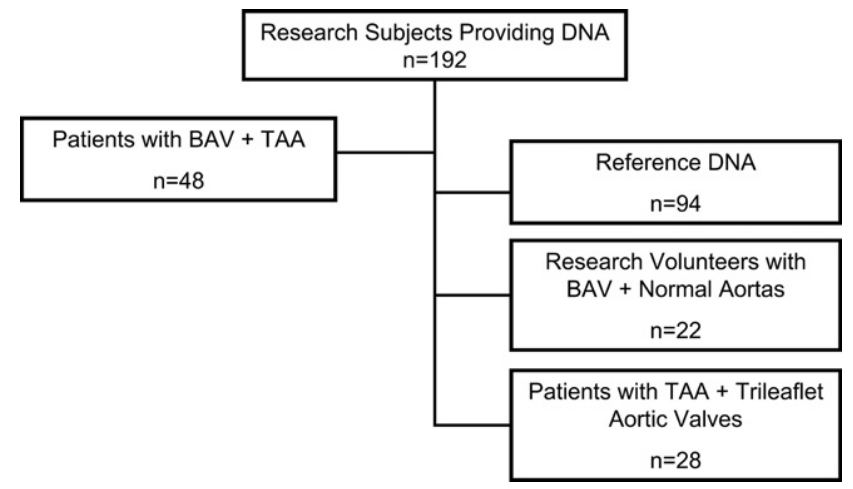

Figure 1. Flow chart of research subjects. DNA was analyzed from 192 subjects, BAV/TAA ( $n=48$ ), BAV with normal aortas ( $n=22$ ), and TAV/TAA ( $n=28$ ). BAV, Bicuspid aortic valve; TAV, trileaflet aortic valve; $T A A$, thoracic aortic aneurysm.

(TAV), as well as a limited number of subjects with BAV and normal aortas $(\mathrm{n}=22)$ or TAV and TAA $(\mathrm{n}=28)$.

\section{Materials and Methods}

After institutional review board approval, written informed consent was obtained for all 192 unrelated subjects. We performed a targeted mutational analysis of the 4 exons (11, 20, 25 and 29) previously implicated in aortic valve disease ${ }^{16,17}$ in 48 unrelated patients with combined BAV and TAA. Comparisons were made among subjects with BAV and normal aortas $(n=22)$, TAV and TAA $(n=28)$, and reference alleles from control subjects with TAV $(\mathrm{n}=94)$ as seen in Figure 1.

We defined an abnormal aorta as an ascending aorta that measured more than $4.0 \mathrm{~cm}$ at its greatest diameter. DNA for all BAV and TAA subjects was extracted either from peripheral lymphocytes using the Puregene DNA extraction kit (Gentra, Inc, Minneapolis, Minn) or from aortic tissue obtained at the time of surgery (Wizard SV genomic DNA purification system; Promega Corp, Madison, Wis). Genomic DNA for 94 subjects with TAV was acquired from patients residing in Olmsted County, Minnesota, participating in an epidemiologic heart disease study. ${ }^{18}$ Echocardiograms were available for control subjects to confirm valve phenotype.

Using polymerase chain reaction (PCR), DHPLC, and direct DNA sequencing, we performed a targeted mutational analysis of the 4 exons $(11,20,25$, and 29) of NOTCH1 previously implicated in BAV, ${ }^{16,17}$ as previously described. ${ }^{19} \mathrm{PCR}$ amplification primers were designed with Oligo software (Molecular Biology Insights, Inc, Cascade, Colo). PCR primers are shown in Table 1. In brief, DHPLC is a sensitive method used to elucidate unknown gene mutations. It is based on thermal energy found in the formation and separation of double-stranded DNA fragments containing a mismatch in the base pairing between the "wild type" and "mutant," or heteroduplex, DNA strands. PCR-amplified DNA is injected onto a solid phase column that is heated to a specific temperature (individually optimized for each unique PCR product), which allows for partial denaturing of the DNA sequence of interest. A linear acetonitrile gradient based on the size of the 
TABLE 1. Primers for screened NOTCH1 exons

\begin{tabular}{lllc}
\hline Primer sequences & \multicolumn{1}{c}{ Forward primer } & \multicolumn{1}{c}{ Reverse primer } & Fragment size (bp) \\
\hline Fragment & & & 400 \\
Notch1-11 & TCC TCA GGT TGG CAC AGG TG & CAC GTG TCG GTC AGT CCT CA & 239 \\
Notch1-20 & TGG CAG ATG TGC GTT CTG AG & AGC AGG TTA CCT TGT CGC TG & 387 \\
Notch1-25A & AGG AGA GCG GTG GCA CTG CTG & CCC CGA AGC TGT AGT CCA GGA & 331 \\
Notch1-25B & TGT GAG CCC ACA TCC GAG AGC & GGC TGA GTT GCA CTG GCT GTC & 251 \\
Notch1-25C & GCT CCC TCA ACT TCA ATG ACC & CCT GAG CAG AGC CTT AGA ACT G & 257 \\
Notch1-29 & GCC CAC CTC CCA CGC CAG & GGG AGT GAG CAG AGC CTG TCA & \\
\hline
\end{tabular}

PCR product is applied to the column to flush the DNA strands and send the PCR products through an ultraviolet detector, resulting in a chromatogram showing the sample's elution profile. Since heteroduplex (mutant sequences) species are less thermodynamically stable than homoduplexes (normal sequences), these double-stranded complexes will begin to unravel at the elevated temperature and elute from the column sooner then their homoduplex or "wild-type" counterparts. As a result, "mutant" DNA yields a DHPLC profile that is different from the "wild type" profile, enabling the detection of mutation harboring samples whose precise genetic mutation is then established by direct DNA sequencing.

To be regarded as a putative BAV/TAA-susceptibility variant, the following criteria had to be met. First, the genetic variant had to be a nonsynonymous variant (synonymous single-nucleotide polymorphisms were excluded from consideration), resulting in a predicted change in the translated amino acid. Second, the variant had to involve a highly conserved residue. Finally, the variant had to be absent among 188 reference alleles from control subjects with echo-confirmed TAV. Mutations were annotated by the single letter nomenclature whereby A1343V, for example, designates a DNA alteration producing a missense mutation involving a substitution of an alanine (A) by valine (V) at amino acid position 1343.

An in silico analysis was performed with PredictProtein (a Web-based tool found at www.predictprotein.org) to predict effect on secondary protein structure. ${ }^{20}$

Differences in the proportion of patients with DNA sequence variations among groups were statistically analyzed with the Fisher exact test. Patient demographic data were analyzed with the Student $t$ test, Wilcoxon rank sum, or Fisher exact test where appropriate.

\section{Results}

A targeted mutational analysis of $\mathrm{NOTCH1}$ was performed on 192 unrelated patients with BAV/TAA $(n=48)$, BAV and normal aorta $(n=22)$, TAV/TAA $(n=28)$, or TAV and normal aorta $(\mathrm{n}=94)$ who served as reference alleles. Table 2 summarizes the demographic, clinical, and pathologic data for BAV/TAA cases $(n=48)$ and those with TAV/TAA $(\mathrm{n}=28)$. Compared with TAV/TAA subjects, the BAV/TAA patients were more likely male (79\% vs $54 \% ; P=.04$ ), had more hypercholesterolemia (40\% vs $14 \% ; P=.02)$, and had smaller aortic aneurysms (50 \pm 7 vs $57 \pm 9 \mathrm{~mm} ; P<.01)$. BAV disease for the BAV/TAA cohort demonstrated fusion of the right/left coronary cusps in $94 \%$ of cases and the presence of raphé and valve calcification in $85 \%$ and in $64 \%$ of cases, respectively.

Clinical data were not available for the majority of patients with isolated BAV $(n=22)$ inasmuch as most were volunteers participating in a study of kindreds with BAV whose evaluation consisted of only limited 2-dimensional echocardiography to assign valve phenotype in addition to donating DNA. Similarly, limited clinical and demographic data were available for the 94 subjects whose DNA was used as reference alleles inasmuch as they were recruited from an epidemiologic heart disease study. Available demographic data for the entire cohort $(n=2,031)$, from which our 94 subjects were sampled, are $48 \%$ male, mean age $62 \pm 11$ years, and mean body mass index $28.4 \pm$ $5.3 \mathrm{~kg} / \mathrm{m}^{2}$.

Overall, 4 unique nonsynonymous (3 novel) variants were identified in 5 of $48(10.4 \%)$ patients with BAV/TAA compared with only 3 of $144(2.1 \%)$ patients with normal aortas (2/94 reference DNA with trileaflet valves, 1/28 TAV/TAA, and 0/22 with BAV/normal aortas; $P=.02$ ). Table 3 summarizes the clinical characteristics and phenotype of the NOTCH1 mutation-positive patients with BAV/TAA. Of the 5 patients ( 3 men, average age $52 \pm$ 12 years, average aortic dimension $50 \pm 5 \mathrm{~mm}$ ) with NOTCH1 variants, 3 had fusion of the right and left coronary cusps, 3 presented with raphé, 4 underwent AVR, and 2 had evidence of aortic valve calcification. Only 1 of these 5 patients had a family history of either BAV or TAA (patient 1, Table 3). This patient, a 70year-old woman with BAV and a 57-mm ascending aorta, had a family history of both BAV and TAA. We identified both polymorphisms, R1350L and P1377S, in this patient. No other patients with DNA sequence variants had family histories of either BAV or TAA.

Two novel NOTCH1 missense mutations, A1343V and P1390T, involved conserved residues localized to epidermal growth factor-like domains and were absent in 188 reference alleles derived from relatively age- and sexmatched volunteers with echo-confirmed normal TAV (Figure 2). The A1343V mutation was identified in a 41-year-old woman who underwent AVR for a stenotic, 
TABLE 2. Demographic, clinical, and pathologic characterization of patients with BAV/TAA, TAV/TAA, or isolated BAV

\begin{tabular}{|c|c|c|c|c|}
\hline Patient characteristics & BAV/TAA (n = 48) & TAV/TAA $(n=28)$ & BAV + normal aortat $(n=22)$ & $P$ valuef \\
\hline \multicolumn{5}{|l|}{ Demographic data } \\
\hline $\mathrm{Age}^{*}$ & $53 \pm 12$ & $61 \pm 18$ & $56 \pm 17$ & .05 \\
\hline Gender ( $\%$ male) & 79 & 54 & 64 & .04 \\
\hline Comorbiditiest & $\%$ & $\%$ & & \\
\hline Hypertension & 46 & 39 & $\mathrm{~N} / \mathrm{A}$ & .6 \\
\hline Hypercholesterolemia & 40 & 14 & $\mathrm{~N} / \mathrm{A}$ & .02 \\
\hline Smoking & 25 & 25 & $\mathrm{~N} / \mathrm{A}$ & 1 \\
\hline Diabetes & 8 & 0 & $\mathrm{~N} / \mathrm{A}$ & .3 \\
\hline \multicolumn{5}{|l|}{ Family history } \\
\hline Family history of BAV & 13 & 22 & & .4 \\
\hline Family history of TAA & 6 & 18 & & .3 \\
\hline \multicolumn{5}{|l|}{ Bicuspid valve disease (\%) } \\
\hline Cusp fusion (\% R/L) & 94 & & & \\
\hline Raphé (\% present) & 85 & & & \\
\hline Calcification ( $\%$ present) & 64 & & & \\
\hline Stenosis & 25 & & & \\
\hline Regurgitation & 38 & & & \\
\hline Mixed & 29 & & & \\
\hline None & 8 & & & \\
\hline \multicolumn{5}{|l|}{ Aortic disease } \\
\hline Aortic dimension $(\mathrm{mm})^{*}$ & $50 \pm 7$ & $57 \pm 9$ & & $<.01$ \\
\hline
\end{tabular}

BAV, Bicuspid aortic valve; TAV, trileaflet aortic valve; TAA, thoracic aortic aneurysm; N/A, not available. *Presented as mean \pm standard deviation. †Clinical data not available for most subjects with BAV + normal aortas. ‡Statistical comparisons were made between BAV/TAA and TAV/TAA groups. $P<.05$ was considered significant.

noncalcified BAV and concomitant graft replacement of an enlarged ascending aorta. In silico analysis of A1343V demonstrated conserved residues that predicted a change in one of the epidermal growth factor-like domains. The P1390T mutation was identified in a 55-year-old man who underwent AVR for a stenotic, calcified BAV and concomitant reduction aortoplasty of an enlarged ascending aorta.

Additionally, 2 nonsynonymous polymorphisms, R1350L and P1377S, were identified in both patients with BAV/TAA and controls. The R1350L substitution was noted in 1 (42\%) of 48 BAV/TAA cases and $1(1 \%)$ of 94 trileaflet controls, while P1377S substitution was observed in $3(6 \%)$ of $48 \mathrm{BAV} / \mathrm{TAA}$ cases, $1(3.6 \%)$ of 28 TAV/TAA, and $1(1 \%)$ of 94 trileaflet controls. Both genetic variants localized to epidermal growth factor-like domain 35 .

Figure 3 is a topographic summary of DNA sequence variants from the targeted $\mathrm{NOTCH}$ l analysis demonstrating both the missense mutations (A1343V and P1390T) and polymorphisms (R1350L and P1377S).

TABLE 3. Patient clinical characteristics for BAV/TAA patients with NOTCH1 nonsynonymous variants

\begin{tabular}{|c|c|c|c|c|c|c|c|c|c|}
\hline \multirow[b]{2}{*}{ Patient } & \multicolumn{7}{|c|}{ Clinical characteristics for patients with missense mutations } & \multirow[b]{2}{*}{ Operation } & \multirow[b]{2}{*}{ Mutation } \\
\hline & Gender & $\begin{array}{l}\text { Age } \\
\text { (y) }\end{array}$ & $\begin{array}{l}\text { Valve } \\
\text { status }\end{array}$ & $\begin{array}{l}\text { Cusp } \\
\text { fusion }\end{array}$ & Raphé & Calcification & $\begin{array}{c}\text { Aortic } \\
\text { dimension }(\mathbf{c m})\end{array}$ & & \\
\hline \multirow[t]{2}{*}{1} & $\mathrm{~F}$ & 70 & BAV & Unknown & Unknown & Y & 5.7 & $\begin{array}{l}\text { AVR, graft replacement of } \\
\text { ascending aorta }\end{array}$ & $\mathrm{R} 1350 \mathrm{~L}$ \\
\hline & & & & & & & & & P1377S \\
\hline 2 & M & 47 & BAV & $\mathrm{R} / \mathrm{L}$ & Y & $\mathrm{N}$ & 5.2 & Bentall & P1377S \\
\hline 3 & $\mathrm{~F}$ & 41 & BAV & $\mathrm{R} / \mathrm{L}$ & Y & $\mathrm{N}$ & 4.5 & $\begin{array}{l}\text { AVR, graft replacement of } \\
\text { ascending aorta }\end{array}$ & A1343V \\
\hline 4 & M & 55 & BAV & $\mathrm{R} / \mathrm{NC}$ & $\mathrm{N}$ & Y & 4.5 & AVR, reduction aortoplasty & P1390T \\
\hline 5 & M & 47 & BAV & $\mathrm{R} / \mathrm{L}$ & $Y$ & $\mathrm{~N}$ & 4.9 & Valve-sparing root replacement & P1377S \\
\hline
\end{tabular}

Missense mutations absent in controls are in bold print. $F$, Female; $M$, male; $B A V$, bicuspid aortic valve; TAA, thoracic aortic aneurysm; $R$, right coronary cusp; $L$, left coronary cusp; $N C$, noncoronary cusp; $A V R$, aortic valve replacement. 

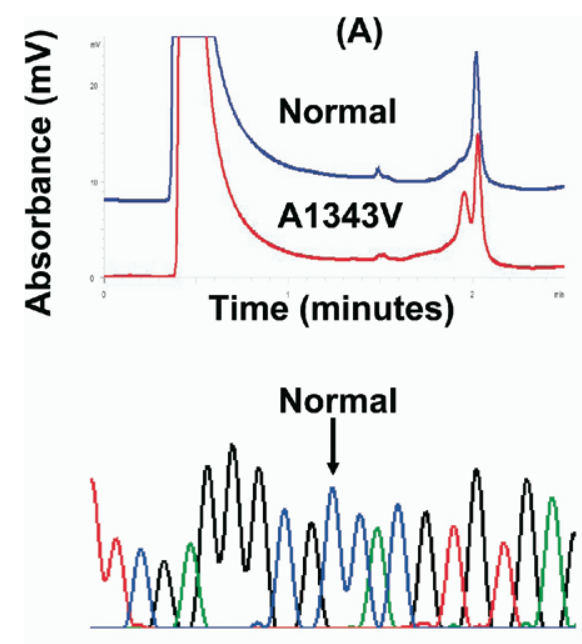

TCGAGGGCGCCACGTGTGA

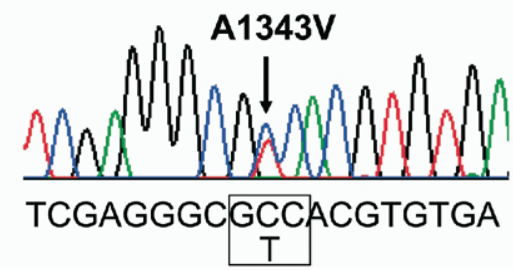

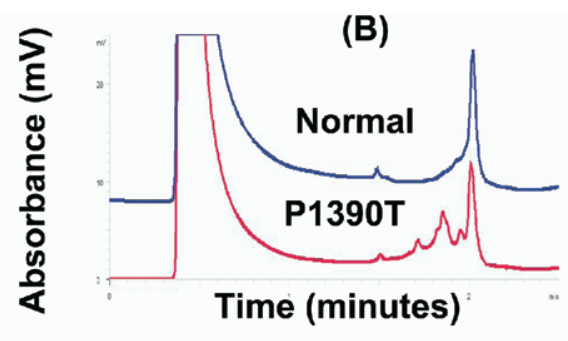
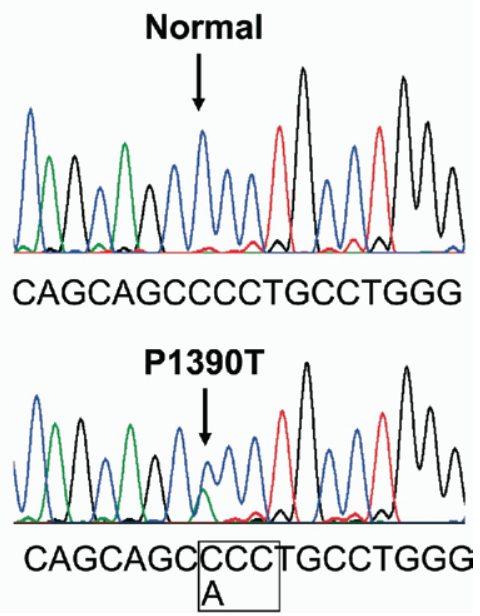

Figure 2. Molecular characterization of novel NOTCH1 missense mutations absent in controls. Depicted are denaturing high performance liquid chromatography (DHPLC) profiles and DNA sequences demonstrating NOTCH1 missense mutations A1343V (A) and B) P1390T.

\section{Discussion}

The principal finding of this targeted mutation analysis of the NOTCH1 gene is the overrepresentation of nonsynonymous sequence variations and the identification of 2 novel missense mutations among patients with combined BAV/ TAA phenotype. These mutations (A1343V and P1390T) were absent in reference alleles and subjects with BAV and normal aortas as well as TAV/TAA. Given the purported roles of the NOTCH1-encoded signaling pathway, we speculated that perhaps genetic variation in NOTCHI may con-

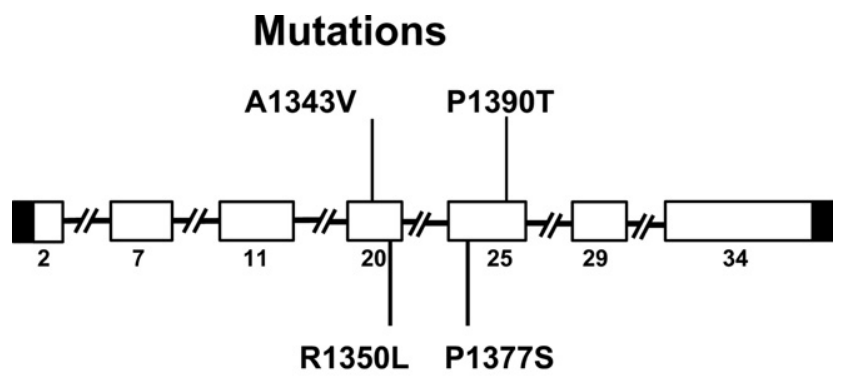

\section{Polymorphisms}

Figure 3. Topographic summary of DNA sequence variants from targeted mutation analysis of NOTCH1 demonstrating missense mutations (A1343V, P1390T) and polymorphisms (R1350L, P1377S). Colors indicate the exons $(11,20,25,29)$ examined in this targeted analysis. fer susceptibility for aortic aneurysmal formation, particularly TAA. The findings of the present study support this notion.

We chose to study the potential association between NOTCHI and patients with BAV/TAA because it has been implicated as a potential susceptibility gene for heritable cardiovascular disease. Garg and colleagues ${ }^{16}$ described NOTCH1 mutations in 2 pedigrees with complex, heterogeneous cardiac disease including BAV, although the status of the ascending aorta was not mentioned. Subsequently, studying nonfamilial cases, Mohamed and coworkers ${ }^{17}$ reported variants in 2 of 48 patients with BAV. Although not the focus of their study, the incidence of NOTCH1 sequence variations was $11 \%(2 / 18)$ among the individuals in their study with concomitant BAV/TAA. Given the size of this gene, we focused our attention on the 4 exons in which NOTCH1 mutations have been reported previously, and this restricted analysis of $\mathrm{NOTCH} 1$ may underestimate the relative contribution of genetic variation in NOTCH1 to the development of TAA.

NOTCH1 is a plausible candidate gene for BAV and TAA. Located at 9q34.3, NOTCH1 encodes for a transmembrane protein that functions as a transcriptional cofactor to activate the Notch signaling pathway, a signaling pathway found in developmental pathways of many organ systems including the human cardiovascular system. ${ }^{21,22}$ Specifically, NOTCH1 has a role in migration of cells from the 
cardiac cushions and cardiac jelly into the conotruncal cushions from which the aortic and pulmonary valves are formed. ${ }^{13-15}$

A role for $\mathrm{NOTCH} 1$ mutations in the pathogenesis of TAA is also plausible. In addition to its role in cardiogenesis and valvulogenesis, the Notch1 signaling pathway has a role in development and maintenance of the aorta and other vessels. ${ }^{13-15}$ Additionally, there is increasing evidence of interaction between the Notch1 pathway and TGF $\beta{ }^{23}$ This is particularly exciting in light of recent reports of TGF $\beta-2$ receptor mutations and TAA in syndromal patients such as those with Loeys-Dietz and Ehlers-Danlos syndromes. ${ }^{7}$ TGF $\beta$ has a known role in regulation of extracellular matrix of aortic tissue. On the basis of the interaction between the Notch1 pathway (valvulogenesis) and TGF $\beta$ (aortic extracellular matrix regulation), we hypothesize that BAV patients with $\mathrm{NOTCHI} \mathrm{mu-}$ tations may be more susceptible to abnormal extracellular matrix regulation and, therefore, aneurysm formation. Although the association between TGF $\beta$ receptor mutations and aortic aneurysm is in its infancy, we believe that exploring an association between $\mathrm{NOTCHI}$ and TGF $\beta$ in patients with nonsyndromal aortic aneurysms (including patients with BAV/ TAA) is certainly intriguing and warranted.

There is increasing interest in the identification of the genetic determinants of aortic valve disease. In 2005, Garg and colleagues ${ }^{16}$ reported NOTCH1 truncation mutations (R1108X and H1505del) in two families with dominantly inherited familial aortic valve disease associated with aortic valve calcification. These deleterious mutations presumably cause haplo-insufficiency and a loss of function phenotype of Notch1 signaling. Among a 5-generation pedigree hosting the truncation mutation, 7 of 9 family members were affected and had calcific aortic stenosis in the setting of either BAV or TAV. Recently, Mohamed and colleagues ${ }^{17}$ identified $\mathrm{NOTCH1}$ missense mutations in approximately $4 \%$ of sporadic cases of BAV.

Limitations of the present study include the heterogeneity in baseline demographics found among our cohorts, as well as the challenge of defining "abnormal" ascending aortic phenotype. BAV/TAA patients had a significantly greater proportion of males and subjects with hypercholesterolemia than did subjects with TAV/TAA. The overall impact this difference makes on interpretation is unknown but is a limitation of the present study. Additionally, defining significant aortic enlargement in patients with BAV remains problematic. One option is to use established nomograms indexed to body mass index, but these are imperfect because measurements may change with fluctuations in body mass. We used a liberal definition of ascending aortic abnormalities at greater than $4.0 \mathrm{~cm}$. If anything, this may have diluted the observation of overrepresentation of DNA sequence variants found in the BAV/TAA cohort. Using a more conservative definition of aortic aneurysm of
$4.5 \mathrm{~cm}$ or larger, the proportion of DNA variants in the BAV/TAA cohort would increase from $10.4 \%$ (5/48) to $12.5 \%(5 / 40)$.

Here, we report the identification of NOTCH1 amino acid altering variants in $10.4 \%$ (5/48) of patients with concomitant BAV/TAA, 0 of 22 BAV subjects without aneurysmal disease, and 1 of 28 patients with TAV/TAA. Although not found to be statistically significant owing to the limited size of our cohort, these data are congruent with recently reported data from Mohamed's group. ${ }^{17}$ They too identified NOTCHI missense mutations in $2(11 \%)$ of 18 patients with sporadic $\mathrm{BAV}$ and concomitant aortic aneurysm and in none of $30 \mathrm{BAV}$ patients without aneurysmal disease.

Taken together, these independent investigations suggest a significant overrepresentation of NOTCH1 missense mutations in BAV/TAA (7 of 66 compared with 0 of 52; $P=.01)$. These observations may offer insight into predicting which patients with BAV are at risk for the development of TAA. To be sure, these studies warrant further investigation into the role of $\mathrm{NOTCHI}$ missense variants in the pathogenesis of aneurysmal associated valve disease. Whether these NOTCH1 missense variants lead to a "gain" or "loss" of function phenotype will require further functional characterization.

Exploration of additional NOTCHI exons should be included in future investigations into the role of $\mathrm{NOTCHI}$ in the extracellular matrix of the ascending aorta.

\section{References}

1. Warnes CA. The adult with congenital heart disease: born to be bad? $J$ Am Coll Cardiol. 2005;46:1-8.

2. Ward C. Clinical significance of the bicuspid aortic valve. Heart. 2000;83:81-5.

3. Russo CF, Mazzetti S, Garatti A, Ribera E, Milazzo A, Bruschi G, et al. Aortic complications after bicuspid aortic valve replacement: long-term results. Ann Thorac Surg. 2002;74:S1773-6; discussion S1792-9.

4. Rankin JS, Hammill BG, Ferguson TB Jr, Glower DD, O'Brien SM, DeLong ER, et al. Determinants of operative mortality in valvular heart surgery. J Thorac Cardiovasc Surg. 2006;131:547-57.

5. Clementi M, Notari L, Borghi A, Tenconi R. Familial congenital bicuspid aortic valve: a disorder of uncertain inheritance. Am J Med Genet. 1996;62:336-8.

6. Cripe L, Andelfinger G, Martin LJ, Shooner K, Benson DW. Bicuspid aortic valve is heritable. J Am Coll Cardiol. 2004;44:138-43.

7. Loeys BL, Schwarze U, Holm T, Callewaert BL, Thomas GH, Pannu $\mathrm{H}$, et al. Aneurysm syndromes caused by mutations in the TGF-beta receptor. $N$ Engl J Med. 2006;355:788-98.

8. Guo D, Hasham S, Kuang SQ, Vaughan CJ, Boerwinkle E, Chen H, et al. Familial thoracic aortic aneurysms and dissections: genetic heterogeneity with a major locus mapping to 5q13-14. Circulation. 2001;103:2461-8.

9. Vaughan CJ, Casey M, He J, Veugelers M, Henderson K, Guo D, et al. Identification of a chromosome 11q23.2-q24 locus for familial aortic aneurysm disease, a genetically heterogeneous disorder. Circulation. 2001;103:2469-75.

10. Hasham SN, Willing MC, Guo DC, Muilenburg A, He R, Tran VT, et al. Mapping a locus for familial thoracic aortic aneurysms and dissections (TAAD2) to 3p24-25. Circulation. 2003;107:3184-90. 
11. Pannu H, Tran-Fadulu V, Milewicz DM. Genetic basis of thoracic aortic aneurysms and aortic dissections. Am J Med Genet C Semin Med Genet. 2005;139:10-6.

12. Baron M. An overview of the Notch signalling pathway. Semin Cell Dev Biol. 2003;14:113-9.

13. Timmerman LA, Grego-Bessa J, Raya A, Bertran E, Perez-Pomares JM, Diez J, et al. Notch promotes epithelial-mesenchymal transition during cardiac development and oncogenic transformation. Genes Dev. 2004; 18:99-115.

14. Alva JA, Iruela-Arispe ML. Notch signaling in vascular morphogenesis. Curr Opin Hematol. 2004;11:278-83.

15. Garg V. Molecular genetics of aortic valve disease. Curr Opin Cardiol. 2006;21:180-4.

16. Garg V, Muth AN, Ransom JF, Schluterman MK, Barnes R, King IN, et al. Mutations in NOTCH1 cause aortic valve disease. Nature. 2005;437:270-4.

17. Mohamed SA, Aherrahrou Z, Liptau H, Erasmi AW, Hagemann C, Wrobel S, et al. Novel missense mutations (p.T596M and p. P1797H) in NOTCH1 in patients with bicuspid aortic valve. Biochem Biophys Res Commun. 2006;345:1460-5.

18. Senni M, Tribouilloy CM, Rodeheffer RJ, Jacobsen SJ, Evans JM, Bailey KR, et al. Congestive heart failure in the community: a study of all incident cases in Olmsted County, Minnesota, in 1991. Circulation. 1998;98:2282-9.

19. Tester DJ, Will ML, Ackerman MJ. Mutation detection in congenital long QT syndrome. In: Wang QK, editor. Cardiovascular disease: methods and protocols. Vol 1. Genetics. Humana Press; Totowa [NJ]. 2006. p. 181-207.

20. Rost B, Yachdav G, Liu J. The PredictProtein server. Nucleic Acids Res. 2004;32:W321-6.

21. Baron M. An overview of the Notch signalling pathway. Sem Cell Dev Biol. 2003;14:113-9.

22. Iso T, Hamamori Y, Kedes L. Notch signaling in vascular development. Arterioscler Thromb Vasc Biol. 2003;23:543-53.

23. Kluppel M, Wrana JL. Turning it up a Notch: cross-talk between TGF beta and Notch signaling. Bioessays. 2005;27:115-8.

\section{JTCVS On-Line Manuscript Submission and Review}

The Journal of Thoracic and Cardiovascular Surgery requires authors and reviewers to submit all new and revised manuscripts and reviews via Editorial Manager. Point your browser to http://jtcvs.editorialmanager.com, log in as author or reviewer (as appropriate), and follow the instructions provided.

To retrieve your username and password, click "Forget your password?" on the Editorial Manager log-in page.

If you have questions or experience problems uploading your manuscript or review, please contact the editorial office:

Telephone: $215-762-1854$

E-mail: jtcvs@drexelmed.edu 